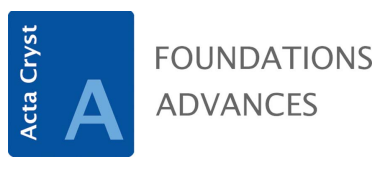

ISSN 2053-2733

\section{Atomic and Molecular Physics. A Primer. By Luciano Colombo. IOP Science, 2019. Ebook, pp. 219. ISBN 978-0-7503-2260-7.}

\author{
Claude Millot*
}

Université de Lorraine, CNRS, LPCT, Nancy, France. *Correspondence e-mail: claude.millot@univ-lorraine.fr

This book is a good introduction to quantum mechanics applied to atomic and molecular systems. It is composed of three parts that present the concepts of quantum mechanics, their applications to atomic physics and to molecular physics. The section about atomic physics constitutes roughly half of the book. In addition, eight appendices give substantial details about formal derivations and numerical calculations. As the title indicates, this book is a primer and the bibliography, which refers to more than 30 textbooks, will allow the reader to delve further into the subject.

The first part presents a historical and conceptual review of the constitution of atoms and the emergence of quantum physics. The first chapter explores the nature of the atom, the quantum nature of physical laws governing atomic spectra and black-body radiation, and wave-particle duality. It ends with the introduction of the wavefunction associated with a particle and the time-independent Schrödinger equation. The second chapter presents the mathematical aspects of quantum mechanics. It explains the notions of wavefunction, quantum operators associated with physical properties, time evolution of the wavefunction, and symmetry of the wavefunction. It concludes with the presentation of the very useful time-independent and time-dependent perturbation techniques of approximation.

The second part explains how quantum mechanics describe atomic systems. Chapter 3 is devoted to the determination and description of the stationary states of the hydrogen atom and the hydrogenoid ions. Then, the magnetic moment as well as the interaction of the atom with a uniform magnetic field (the Zeeman effect) are discussed. The author, after having described the action of a non-uniform magnetic field (the Stern-Gerlach experiment), introduces the notion of spin. The next part of the chapter deals with the analysis of spin-orbit coupling as well as with the fine and hyperfine structure of the energy spectrum. This long chapter ends with the description of how an atom interacts (i) with a uniform magnetic field taking the spin into account (the anomalous Zeeman and Paschen-Back effects) and (ii) with a static electric field (the Stark effect). The following chapter studies the interaction of atoms with electromagnetic fields within a semi-classical framework in which the atom is treated quantum mechanically whereas the electromagnetic field is treated classically by Maxwell equations. Absorption and emission processes are discussed and the microscopic theory of Einstein coefficients is presented. The chapter ends with a discussion of the selection rules for transitions as well as the LASER effect. The last chapter of this part is about multi-electron atoms. In this case, the electron-electron interactions and the symmetry of the wavefunction including the spin - should be taken into consideration in order to get accurate models. The author starts with a simple central-field approximation, which leads to the main features of the periodic table of the elements. He then elaborates on the more sophisticated Hartree, Hartree-Fock and configuration interaction approaches. The chapter continues with the analysis of the energy spectra of multi-electron atoms, which includes the couplings between individual electronic orbitals and spin momenta. This leads to the L-S (light atom, small spin-orbit coupling) and J-J (heavy atom, strong spin-orbit coupling) coupling schemes. The selection rules and the interaction of a multi-electron atom with a static uniform magnetic field end this chapter.

The third part deals with the description of molecules composed of two or more atoms linked by chemical bonds. Chapter 6 discusses the nature of molecules as well as the representation and the determination of a molecular wavefunction. First, the Born- 
Oppenheimer approximation technique is presented in order to legitimate the separation between the nuclei and the electron motions. Consequently, the molecular wavefunction is viewed as a product of a nuclear wavefunction and an electronic one. Then, the concepts of ionic bond and covalent bond are presented. The ionic bond is illustrated by the example of the $\mathrm{NaCl}$ molecule and the covalent bond by the $\mathrm{H}_{2}$ molecule. In fact, the $\mathrm{NaCl}$ molecule should not be presented as an exact prototype of ionic bonds. Indeed, in this molecule, as well as in any kind of molecule, the bond is not $100 \%$ ionic; the bond is fully ionic in crystals only: a more specific explanation would have been welcome. Chapter 7 deals with molecular vibrations and rotations, starting with the quantum treatment of diatomic molecules within the models of the rigid rotor and harmonic oscillator. It also discusses the energy spectra and selection rules for vibrational (infrared) and rotational (far-infrared or microwave) spectroscopies as well as Rayleigh and Raman vibrational and rotational scattering. Moreover, the author gives a short presentation of the more complex quantum description of the rotation and vibration of polyatomic molecules. In this part, he introduces the powerful model of vibrational normal modes. The last chapter concerns the general description of the electronic structure of diatomic and polyatomic molecules. The insightful concept of molecular orbitals, which are constructed as linear combinations of atomic orbitals, is presented together with the variational method allowing their optimization. The diagram of molecular orbitals is built for homonuclear diatomic molecules using the powerful concept of molecular symmetry. The variational method is then extended to heteronuclear diatomic molecules. Furthermore, this strategy is applied to polyatomic molecules with a specific restriction: the author used it solely for the atomic orbital containing the unpaired electrons of the atoms within the molecule ( $N$ electrons). Thus, this method would optimize $N$ molecular orbitals by solving a linear system of $N$ equations with $N$ unknowns. Such a restriction is unnecessary. In practice, dealing with all the atomic orbitals and electrons of the valence shells is preferable and not particularly more difficult. The directionality of molecular orbitals is then discussed using the concept of orbital hybridization. This discussion is based on the example of the carbon atom, which is a relevant illustration of the spatial shape of molecular orbitals. However, the argument explaining carbon tetravalence is debatable. It is stated that since the difference in energy between the $2 s$ and $2 p$ states is small, the excited configuration $(2 s)^{1}(2 p)^{3}$ is most likely to be found at room temperature rather than the $(2 s)^{2}(2 p)^{2}$ one. Indeed, experimental results (Haris \& Kramida, 2017) show that the difference in energy between the lowest-energy terms of these two configurations $\left({ }^{3} \mathrm{P}\right.$ for the ground state and ${ }^{5} \mathrm{~S}$ for the excited configuration) is about $4.2 \mathrm{eV}$. If we take the second term of the excited configuration $\left({ }^{3} \mathrm{D}\right)$, the energy difference is around $8.0 \mathrm{eV}$. At $298 \mathrm{~K}, k_{\mathrm{B}} T$ is close to $0.026 \mathrm{eV}$; therefore the probability of the excited configuration is very low. In this chapter, the description of the different hybridization states of the carbon atom opens the door to the field of organic stereochemistry. This last chapter ends with the treatment of the electronic transitions and the FranckCondon principle.

With this book, Professor Colombo gives a very interesting overview of basic atomic and molecular quantum mechanics which would be useful for undergraduate students in physics, chemistry, earth sciences and material sciences. It could also interest graduate students in chemistry and in other fields wishing to become familiar with the world of quantum mechanics. One great quality of this book is that it is very pleasant to read. Moreover, it makes the subject seem easy even when the topic is rather complex. A few mathematical calculations have been skipped; it would have been of interest to find them in the appendices but, overall, the various steps are detailed and very well presented. I am convinced that students will enjoy this book. What's more, it will give them a strong motivation to learn more about this topic.

\section{References}

Haris, K. \& Kramida, A. (2017). ApJS, 233, 16. 